\title{
Evolution of nanoscale interstitial dislocation loops under coupling effect of stress and temperature
}

\author{
Ning Gao ${ }^{1, *}$, Tielong Shen ${ }^{1}$, Richard Kurtz ${ }^{2}$, Zhiguang Wang ${ }^{1}$, Fei Gao ${ }^{3 *}$ \\ ${ }^{1}$ Lboratory of Advanced Nuclear Material, Institute of Modern Physics, LanZhou 730000, PR \\ China \\ ${ }^{2}$ Pacific Northwest National Laboratory, P. O. Box 999, Richland, WA 99352, USA \\ ${ }^{3}$ Department of Nuclear Engineering and Radiological Sciences, University of Michigan, Ann \\ Arbor, MI 48109, USA \\ *corresponding. ning.gao@impcas.ac.cn and gaofeium@umich.edu
}

Keywords: dislocation loop, coupling effect, decomposition, non-conservation of Burgers vector, molecular dynamics (MD).

\begin{abstract}
The properties of nano-scale interstitial dislocation loops under the coupling effect of stress and temperature are studied using atomistic simulation methods and experiments. The decomposition of a loop by the emission of smaller loops is identified as one of the major mechanisms to release the localized stress induced by the coupling effect, which is validated by the TEM observations. The classical conservation law of Burgers vector cannot be applied during such decomposition process. The dislocation network is formed from the decomposed loops, which may initiate the irradiation creep much earlier than expected through the mechanism of climb-controlled glide of dislocations.
\end{abstract}


When materials are operated under the irradiation environment, the formations of self-interstitial atoms (SIAs), SIA-clusters and SIA-loops induced by the interaction between high energy particles and atoms in materials are found to play an important role in understanding different radiation damage processes ${ }^{1,2,3,4,5,6,7,8,9,10,11}$. Under irradiation, the clustering and growth of supersaturated SIAs form SIA dislocation loops, which can be observed by transimssion electron microscopy (TEM) when its size reaches to nano-scale. In BCC iron, two Burgers vectors of these loops are confirmed: $1 / 2<111\rangle$ and $\langle 100\rangle$, with different properties ${ }^{4,6,7,9,10}$, which are usually explored without considering the stress or strain effect. However, under irradiation (e.g. creep condition), both temperature and stress affect the evolution of dislocation loop in a coupled way, which has not fully studied until now. Recently, the TEM observation of the in-situ ion irradiation has been widely used to understand the radiation damage ${ }^{9}$. By analyzing such experimental process ${ }^{9}$, we notice that the high energy in-situ irradiation can result in not only the temperature effect but also the possible thermal-stress effect on existed loops in nano-thick TEM samples. The possible free surface effect induced by nano-thick TEM samples may also affect the evolution of loop. Hence, the evolution of dislocation loops is affcetd by a coupling condition of stress and temperature. Except such external stress, the high density of the extended defects (e.g. helium bubbles, precipitates) produced by irradiation would also induce high local stress in materials, which may influence the state of dislocation loops. As one of the extended defects, the state change of SIA dislocation loops under the coupling condition is supposed to influence the mechanical properties of irradiated samples. Thus, all these results lead us to address the following questions: (1) how does the coupling condition of stress and temperature influence the properties of nano-scale dislocation loops in irradiated materials? and (2) what is the detail of the structural evolution under such coupling effect?

Without stress effect, the stable defect configuration of a single interstitial is a $<110>$ dumbbell in $\mathrm{BCC}$ iron $^{12,13,14}$, different from the $\left\langle 111>\right.$ crowdion in other BCC metals ${ }^{15}$. 
When the $\langle 110\rangle$ dumbbell is affected by external stress field, e.g from helium-vacancy cluster, it can transfer to the $<111>$ crowdion with a low energy barrier $(0.3 \sim 0.4 \mathrm{eV})^{16}$. Recent calculation shows the formation energy of simple defects (e.g. dumbbell and crowdion) in BCC iron and tungsten is also affected by external hydrostatic strain and uniaxial strain along the $\langle 100\rangle$ direction of $\mathrm{BCC}$ lattice or axial direction of a defect, that the compressive stress increases the formation energy while the tensile stress results in an opposite trend ${ }^{17,18,19}$. MD results show that the interstitials in BCC iron can form a dislocation loop along the $<111>$ direction when the number of interstitials is more than $4 \sim 5$, even the initial state of SIAs in simulations was set as a parallel $\langle 110\rangle$ cluster $^{6}$. Thus, it is reasonable to consider, based on these state changes, that the coupling effect of temperature and stress would also induce a SIA-loop to migrate on free energy surface between different states. In this paper we will address such coupling effect on the states of nano-scale SIA dislocation loops by simulation and experimental methods to understand the properties of these nano-scale loops, which may be significant for further studying the radiation damage and developing radiation resistance materials.

The Ackland-2004 empirical Fe potential ${ }^{20}$ is mainly employed in the present work. The initial $1 / 2<111>$ dislocation loops are constructed by inserting three extra atomic layers on a $\{111\}$ atomic plane along the $<111>$ direction, in which its Burgers vector is perpendicular to the habit plane. The high elastic energy produced during the insertion is removed by moving the affected atomic layers related with the insertion according to the calculated displacement fields. The shape of the dislocation loop is determined as a hexagon or a circle according to the experimental observations ${ }^{4,5,6,9}$. The computation box consists of $34 \times 12 \times 21$ unit cells along X[111], Y[112] and Z[1110] directions, respectively, consisting of 51408 atoms in such matrix. The loops contain the number of interstitials from 19 to 151 in the computational boxes. Periodic boundary conditions are employed along the three directions during the simulations. The box with a loop is firstly relaxed with molecular static (MS) method. Then 
the MD simulations are performed with temperatures from 0 to $600 \mathrm{~K}$ in which the velocity direction) or perpendicular to the Burgers vector (along $\mathrm{Y}$ and $\mathrm{Z}$ directions) is applied at constant strain rates at each temperature with the same method desribed in Ref[21]. During the application of strain, the Poisson effect is considered along the directions perpendicular to the uniaxial strain direction, which is suitable for studying the uniaxial stress effect on samples. This method has been extensively and successfully used in the MD simulation of mechanical response in nanocrystal materials ${ }^{20}$. The time-step is chosen to be $1 \mathrm{fs}$. The experiments have also been performed with a 9\%-12\% Cr modified F/M steel (SIMP-steel) by $24 \mathrm{MeV}$ iron irradiation during which the constant force is applied on sample at around $570 \mathrm{~K}$. The initial stress is around $200 \mathrm{MPa}$. The experimental approach and results will be reported in detail elsewhere later. Here, we only discuss the related TEM studies to validate the simulation results.

The stress-strain $(\sigma-\varepsilon)$ curve for the MD box containing a $1 / 2<111>$ loop under the coupling condition is firstly calculated. Without stress, the temperature is found to only induce the change of the habit plane of a loop between $\{111\},\{110\}$ and $\{211\}$ within MD time scale. Under applied stress at a given temperature, the $\sigma-\varepsilon$ curve firstly shows a general feature of ductile material with the stress up to around $20 \mathrm{GPa}$, that is, the stress value increases continuously with the strain. Above $20 \mathrm{GPa}$, the $\sigma-\varepsilon$ curve shows a turning point, which is defined as the ultimate stress point (USP), as shown in Figure 1(a) (black line) for a system containing a 151-SIAs loop. It seems that the USP appears to be related with the configuration evolution of the dislocation loop, as explained in the following section. When the stress is smaller than the USP value for both compressive and tensile cases, the properties of the dislocation loop found to be remained as before, that is, the Bugers vector is still $1 / 2<111>$ and the shape is also unchanged. However, in the region close the dislocation core, a large number of atoms moves to high energy states (potential energy, $\mathrm{E}_{\mathrm{pot}}>-3.85 \mathrm{eV}$ ) under 
tensile stress, but an opposite trend is observed under compressive stress. These results may respectively ${ }^{17,18,19}$.

When the simulations are performed under the coupling condition with temperatures from 150 to $600 \mathrm{~K}$ and stress applied along the Burgers vector, the $\sigma-\varepsilon$ curve also shows the USP as indicated in Figure 1(a). The similar results are observed with the different loop sizes and strain rates. Under the tensile stress, the USP appears with the $\varepsilon$ values around $0.148,0.125$, 0.108 and 0.099 at $150,300,450$ and $600 \mathrm{~K}$, respectively. Under the compressive stress, the temperature has less influence on the USP with the $\varepsilon$ values ranged from -0.091 to -0.054 . Comparing with the results obtained at $0 \mathrm{~K}$, these results indicate that the coupling effect can readily lead to the state change of the system and induce the system to degenerate. The change of the habit plane is observed between $\{111\},\{211\}$ and $\{110\}$ under the coupling effect. After the USP, the stress value decreases rapidly as shown in Figure 1(a). However, neither micro-crack nor necking is occured just before and after the USP for all the cases studied here. Since the loop is the only special configuration in this system, the evolution of loop configuration is considered as a main reason to induce a discontinuity in the $\sigma-\varepsilon$ curves.

In addtion to the stress along the Burgers vector, the cases with the stress perpendicular to the Burgers vector are also studied, as shown in Figure 1(b) (along Y direction). The result with the stress along $\mathrm{Z}$ direction is similar to the case along $\mathrm{Y}$ direction, which is not shown here. Figure 1(b) exhibits that the stress increases with the strain before the USP and rapidly decreases after. The evolution of the loop configuration is also recognized as a main reason for the USP change. Comparing the $\sigma-\varepsilon$ curves with the stresses along X and $\mathrm{Y}$ directions, it is clear that the USP can be easier established with the stress perpendicular than parallel to the Burgers vector under the tensile stress. In this case, the strain value at the USP is in the range from around 0.082 to 0.106 with decreasing temperature. Under the compressive stress, the results are contrary: the stress at the USP has a larger value with the stress perpendicular than 
parallel to the Burgers vector. The strain value at the USP is widely ranged from around -0.109 to -0.081 . Hence, the tensile stress perpendicular to the Burgers vector can more easily change the loop configurations under the coupling effect.

The structural evolution of a loop under the coupling effect is then illustrated as following. Under the tensile stress, the configuration of a loop before the USP remains its initial shape and Burgers vector. After the USP, the loop decomposes into several small loops, as shown in Figure 2(a). The central large loop remains the Burgers vector of $1 / 2[111]$, but degenerates into an ellipsoid shape, which is different from the 2D planar loop or other 3D SIA-cluster $\left(\mathrm{C} 15\right.$ Laves phase $\left.^{22}\right)$. Six small loops are symmetrically around the central one with different Burgers vectors: $1 / 2[\overline{1} 11], 1 / 2[1 \overline{1} 1]$ and 1/2[111]. Because the sum of Burgers vectors of these small loops are not equal to the Burgers vector of the original large dislocation loop, the Burgers vector conservation law is not satisfied. This is also confirmed by the TEM observations, as shown in Figure 2 (e) and (f), where the emission of small loops is clearly demonstrated. Following the method suggested in Ref[8], the Burgers vector of loops are all family of $1 / 2<111\rangle$. The similar emission of dislocation loops is also observed at the evolution of a crack tip in $\mathrm{FCC} \mathrm{Cu}{ }^{23}$ and in situ $\mathrm{Kr}$-irradiated $\mathrm{UO}_{2}$ single crystal $^{24}$, which is in fact a way to release high stress concentration. Under the compressive stress, the loop decomposes into two large crossover loops. Both of them form a long-flat rectangle shape, but remains the same Burgers vector of 1/2[111], as shown in Figure 2(b). Temperature decreases the USP without affecting above evolution process. Thus, two different mechanisms of loop decomposition are observed to release the stress concentration under the coupling condition, corresponding to the tensile and compressive stresses, respectively.

After the USP, the applied tensile stress continues to decompose these small loops into even smaller loops and finally to atomic configurations of irregular SIA arrays, as shown in Figure 2(c). A similar result is also found for the compressive stress as shown in Figure 2(d), but with less number of SIA arrays. The state of SIA arrays is recognized by DXA analysis 
included in Ovito ${ }^{25}$ as dislocation networks. It is of interest to note that both $1 / 2<111>$ and the dislocation networks from loops generally consider to be formed only when the dislocation loops grow to a critical size and intersect with each other ${ }^{26}$. However, the present results indicate that the decomposition of the radiation-induced nanoscale loops could also result in the dislocation network under the coupling condition of stress and temperature. Since the climb-controlled glide (CCG) irradiation creep can be described by: $\frac{\mathrm{d} \varepsilon_{i j}}{\mathrm{dt}}=e_{i m n} \rho<$ $V_{m} t_{n} b_{j}>$, where $\rho$ is the density of dislocations and $\mathbf{V}$ is the dislocation velocity ${ }^{27}$, the above results imply the CCG mechanism may take place much earlier than expected. After the formation of dislocation network, the stage of nucleation and motion of dislocations has been reached in a single crystal, allowing the system to slip at a much lower stress, which may be a reason why the stress value decreases rapidly after the USP in the stress-strain curve.

The evolution of dislocation loops under the stress perpendicular and parallel to the Burgers vector is different. When the tensile stress is perpendicular to the Burgers vector, the dislocation loop firstly changes its habit plane between $\{111\},\{112\}$ and $\{110\}$. Under the compressive stress, the new habit planes are also observed: when the stress is along $Z[\overline{1} 10]$,

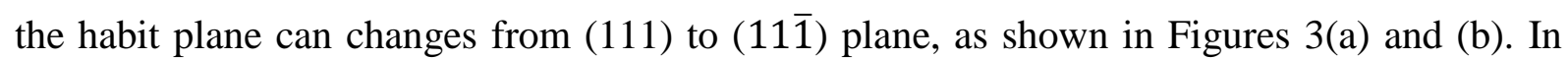
addition, another habit plane, (100), is observed with the stress along the $\mathrm{Y}[11 \overline{2}]$ direction. In both the cases, the shape of loops is changed from a circular to an elliptical shape (Figure 3). The area of these loops increases with the compressive stress, which is also observed for the loops formed in a nanopillar under stress ${ }^{28}$. Another interesting result is that all these habit planes have the same rotation axis along the $Z[\overline{1} 10]$ direction, which is not the same as expected that the stress along $Z[\overline{1} 10]$ would induce the loop rotation around the $Y[11 \overline{2}]$ direction. The possible reason is that if the rotation occurrs around $\mathrm{Y}$ with the stress along the $\mathrm{Z}$ direction, the rotation angle could be $90^{\circ}$, larger than the rotation angle of $48.19^{\circ}$ around $\mathrm{Z}$. 
Thus, when the formed interstitial dislocation loop is affected by the local compressive stress, the component of stress with the direction perpendicular to the Burgers vector can induce the rotation of the loop to form a large elliptical configuration. After the UPS, with the continuous perpendicular tensile stress, the loop firstly changes to a rectangular shape and then spontaneously emits two symmetrical loops located on a (11) plane, as shown in Figure 4(a). Under the continuous perpendicular compressive stress after UPS, a large dislocation loop also emits two small dislocation loops located on (111) and (1111) planes, but the elliptical shape remains unchanged (Figure 4(b)). In both the cases, the emission of loops following the slip systems in BCC metals is a main path for loops to release highly concentrated stress. After the decomposition, the slip of the system is also triggered, which again greatly decreases the stress for futher deformation, as demonstrated in Figure 1(b).

In conclusion, the response of a SIA dislocation loop to the coupling effect of stress and temperature in BCC iron is studied using atomic-level simulations and experiments. The stress-strain curve under a uniaxial stress has been deteremined at different temperatures, and the decomposition of a loop is responsible for the observed USP on the stress-strain curve. When the stress is along the Burgers vector, the tensile stress induces the decomposition of a loop and the compressive stress results in the decomposition and reformation of a large flat rectangular loop. When the stress is perpendicular to the Burgers vector, the emission of small loops and the formation of a large elliptical loop are observed. These results are consistent with the TEM observations. The non-conservation of the Burgers vector during the decomposition process implies that a nano dislocation loop should be regarded as a special phase which transformation can occur by overcoming an energy barrier. The formation of dislocation network from the decompsed loops initiates the irradiation creep much earlier than expected through a climb-controlled glide mechanism.

\section{Acknowledgements}


This work is supported by National Natural Science Foundation of China (Project Nos 11375245,11675230 and 91426301). RJK acknowledge the support of the Office of Fusion Energy Sciences, U.S. Department of Energy under Contract DE-AC05-76RL01830 


\section{References}

1. B. C. Master, Nature 200 (1963) 254.

2. S. Zinkle, B. Singh, J. Nucl. Mater. 351 (2006) 269.

3. J. Marian, B. D. Wirth, J. M. Perlado, Phys. Rev. Lett. 88 (2002) 255507.

4. K. Arakawa, M. Hatanaka, E. Kuramoto, K. Ono, H. Mori, Phys. Rev. Lett. 96 (2006) 125506.

5. H. Xu, R. E. Stoller, Y. N. Osetsky, D. Terentyev, Phys. Rev. Lett. 110 (2013) 265503.

6. J. Chen, N. Gao, P. Jung, T. Sauvage, J. Nucl. Mater. 441 (2013) 216.

7. S. L. Dudarev, R. Bullough, P. M. Derlet, Phys. Rev. Lett. 100 (2008) 135503.

8. J. Chen, P. Jung, W. Hoffelner, H. Ullmaier, Acta Mater. 56 (2008) 250.

9. K. Arakawa, T. Amino, H. Mori, Acta Mater. 59 (2011) 141.

10. Z. Yao, M. Jenkins, M. Hernandez-Mayoral, M. Kirk, Philos. Mag. 90 (2010) 4623.

11. D. Terentyev, G. Monnet, P. Grigorev, Scripta Mater. 69 (2013) 578.

12. P. Ehrhart, K. H. Robrock, H. R. Schober, In Physics of Radiation Effects in Crystals, R. Johnson, A. N. Orlov (eds.), vol. 13, chap. 1, 63 (Elsevier, Amsterdam, 1986).

13. C. Domain, C. S. Becquart, Phys. Rev. B 65 (2001) 024103

14. C. C. Fu, F. Willaime, P. Ordejon, Phys. Rev. Lett. 92 (2004)175503.

15. D. Nguyen-Manh, A. P. Horsfield, S. L. Dudarev, Phys. Rev. B 73 (2006) 020101.

16. N. Gao, H. V. Swygenhoven, M. Victoria, J. Chen, J. Phys. Condens. Matter 23, 442201 (2011).

17. D. Wang, N. Gao, W. Setyawan. R. J. Kurtz, Z. G. Wang, X. Gao, W. H. He, L. L. Pang, Chin. Phys. Lett. 33, (2016) 096102.

18. Z. Chen, N. Kioussis, N. Ghoniem, D. Seif, Phys. Rev. B 81, (2010) 094102.

19. D. Wang, N. Gao, Z. G. Wang, X. Gao, W. H. He, M. H. Cui, L. L. Pang, Y. B. Zhu, Nucl. Instr. Meth. Phys. Res. B 384, (2016) 68

20. G. J. Ackland, M. I. Mendelev, D. J. Srolovitz, S. Han, A. V. Barashev, J. Phys. Condens. Matter 16, (2004) S2629

21. E. Bitzek, C. Brandl, P. M. Derlet, H. Van Swygenhoven, Phys. Rev. Lett. 100 (2008) 235501.

22. M. C. Marinica, F. Willaime, J. P. Crocombette, Phys. Rev. Lett. 108, (2012) 025501. 
23. T. Zhu, J. Li, S. Yip, Phys. Rev. Lett. 93 (2004) 025503.

24. L. F. He, M. Gupta, C. A. Yablinsky, J. Gan, M. A. Kirk, X. M. Bai, J. Pakarinen, T. R. Allen, J. Nucl. Mater. 443, 71 (2013)

25. A. Stukowski, Model. Simul. Mater. Sci. Eng. 18 (2010) 015012.

26. N. M. Ghoniem, G. Kulcinski, Rad. Eff. 39 (1978) 47.

27. T. Mura, Int. J. Eng. Sci., 1 (1963) 371

28. L. Huang, Q. J. Li, Z. W. Shan, J. Li, J. Sun, E. Ma, E. Nat. Commun. 2 (2011) 547 


\section{Figure Caption}

Figure 1: Stress-strain curve for a system containing 151-SIA loop at different temperatures with the stress along (a) X [111] direction and (b) Y[112] direction.

Figure 2: Configurations of a loop after the USP under (a) tensile and (b) compressive

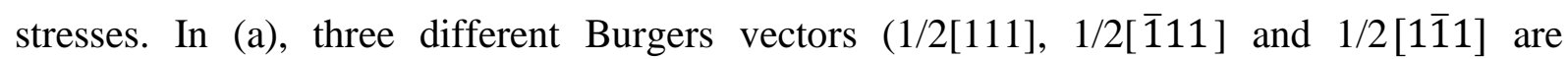
indicated for the different decomposed small loops. The configurations of dislocation network are shown in (c) under tensile stress and (d) under compressive stress after the decomposition. The green and pink lines are dislocations with the Burgers vectors of $1 / 2<111\rangle$ and $\langle 100\rangle$, respectively. The TEM imgaes showing the emission of a loop under the tensile stress in (e) and (f) under the bright and dark fields, respectively.

Figure 3: Configurations of a loop at the initial state and under compressive stress. (a) and (b) are results viewed along the $\mathrm{Z}$ [110] and $\mathrm{Y}[11 \overline{2}]$ dierction, respectively. The gray spheres indicate high energy atoms at the dislocation core without applied stress as a reference state of the loop. The inserted image in (a) shows the initial circle shape. The compressive stress is applied along the [1]10] direction. The elliptical shape of the loop under the compressive stress is indicated by colored atoms and marked by the red line in (b).

Figure 4: Configurations of a loop under (a) tensile and (b) compressive stress after the USP, where the stress is perpendicular to the Burgers vector. In (a), the central loop changes to a rectangular shape and the two loops with a circular shape are emitted under the tensile stress, which are located on a (11) $)$ plane. In (b), the elliptical shape remains for the central loop under compressive stress, but two small dislocation loops are emitted, which are located on $(\overline{1} 11)$ and (11̄1) planes. 

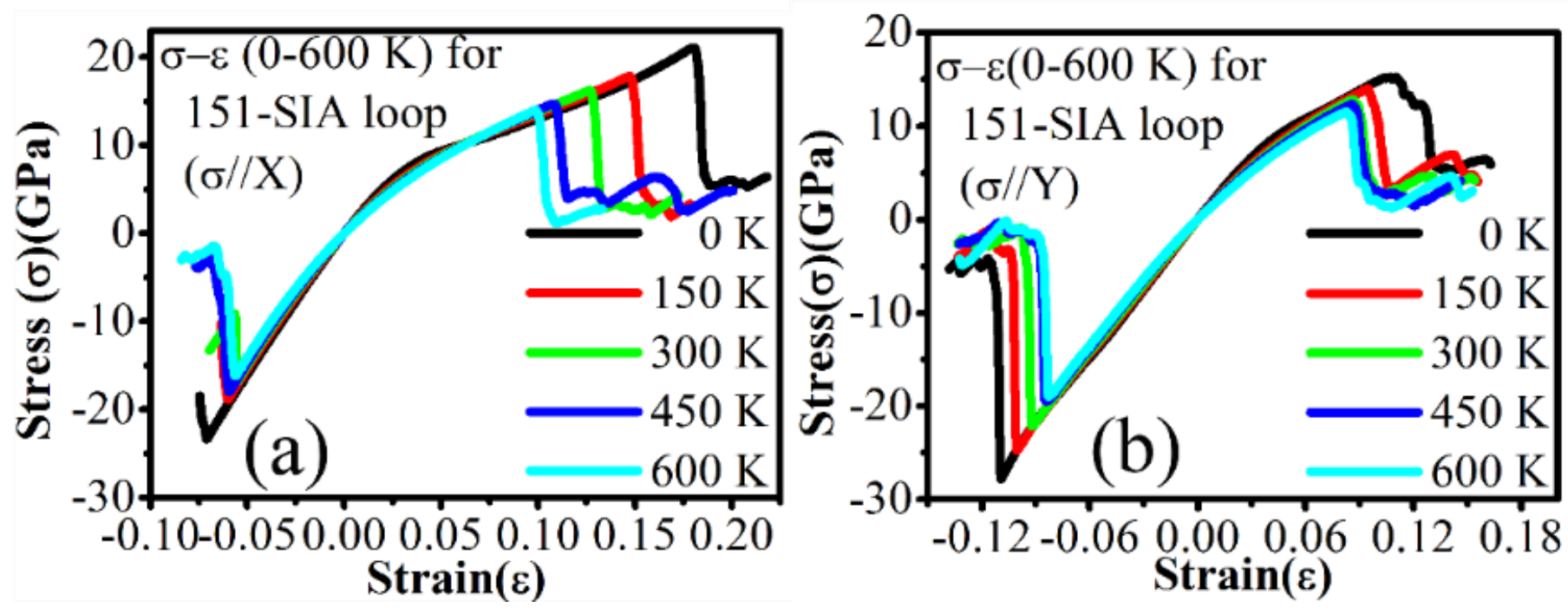


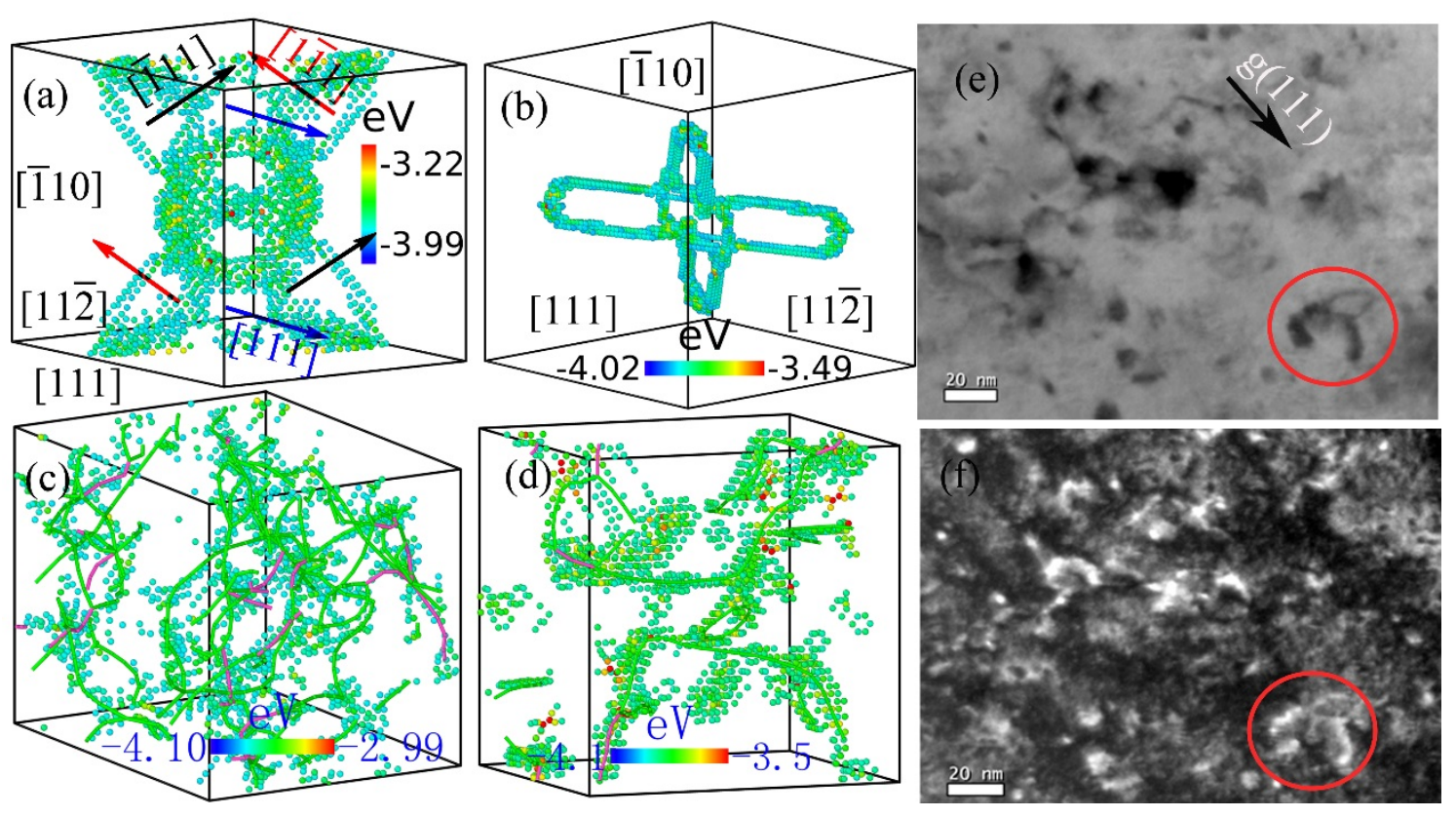




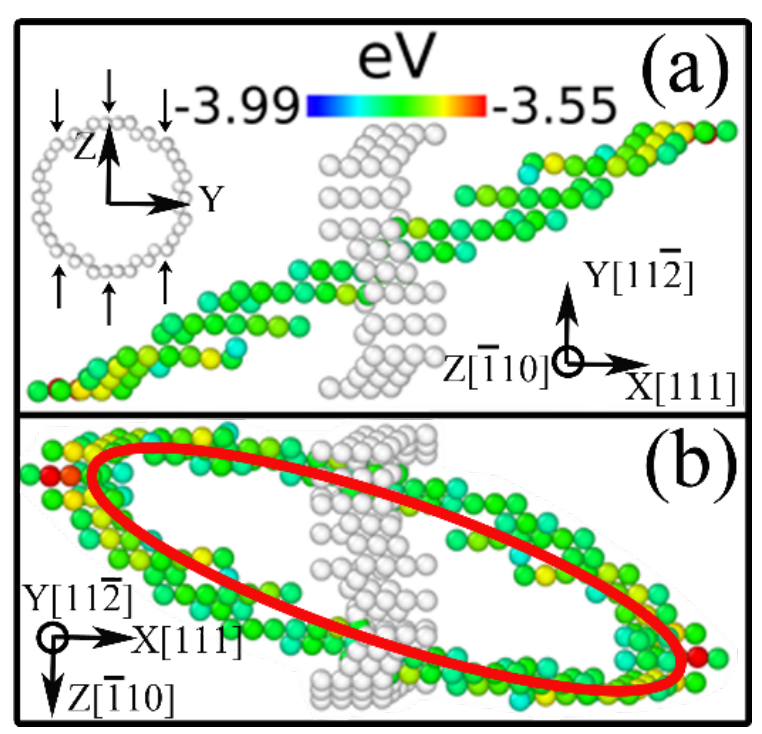




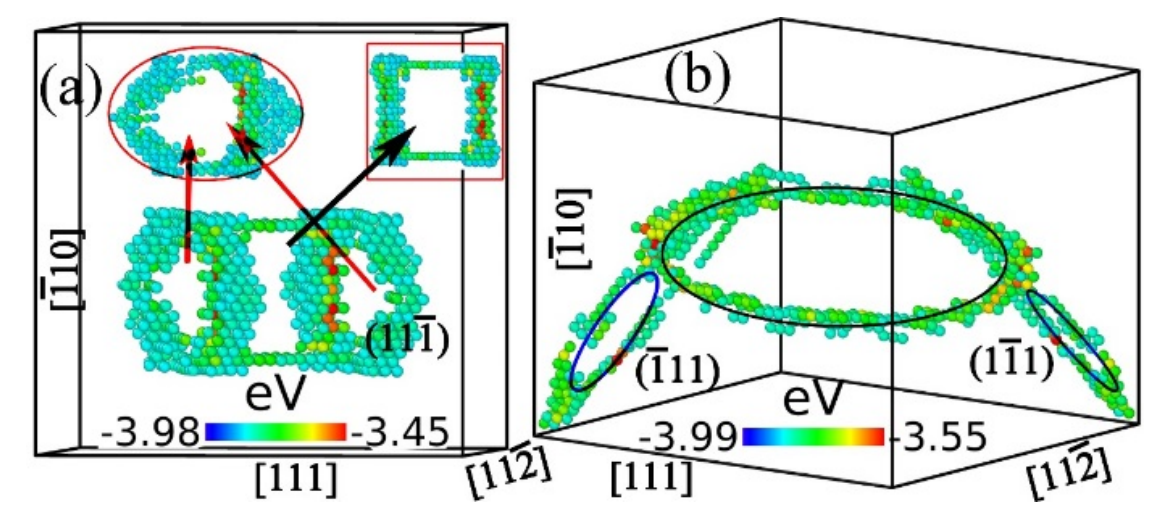

Fig. 4 

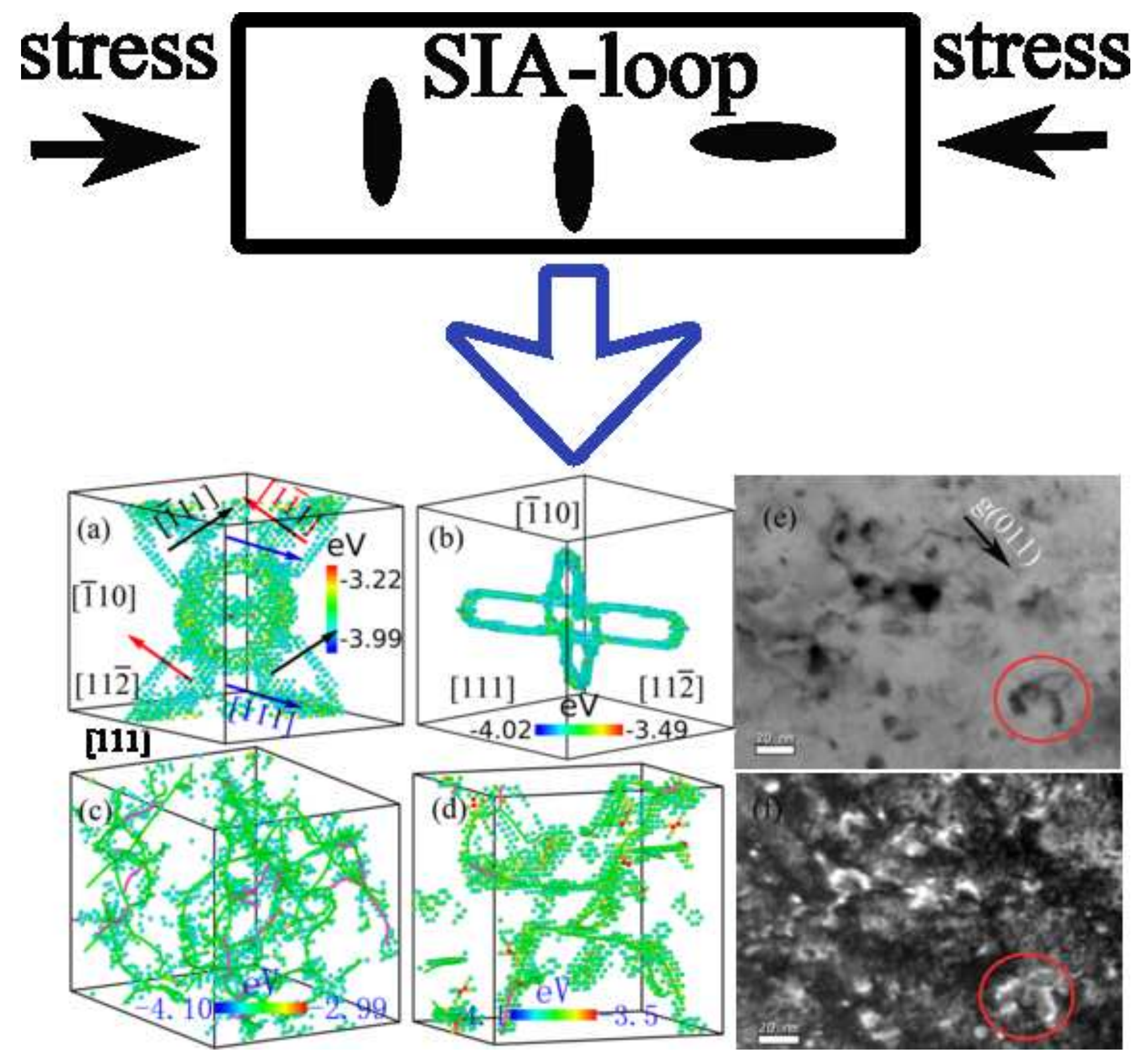